\title{
TU/e EmonOWEN

\section{On the influence of phase noise induced ICI in MIMO OFDM systems}

Citation for published version (APA):

Schenk, T. C. W., Tao, X-J., Smulders, P. F. M., \& Fledderus, E. (2005). On the influence of phase noise induced ICI in MIMO OFDM systems. IEEE Communications Letters, 9(8), 682-684.

https://doi.org/10.1109/LCOMM.2005.1496581

DOI:

10.1109/LCOMM.2005.1496581

Document status and date:

Published: 01/01/2005

\section{Document Version:}

Publisher's PDF, also known as Version of Record (includes final page, issue and volume numbers)

\section{Please check the document version of this publication:}

- A submitted manuscript is the version of the article upon submission and before peer-review. There can be important differences between the submitted version and the official published version of record. People interested in the research are advised to contact the author for the final version of the publication, or visit the $\mathrm{DOI}$ to the publisher's website.

- The final author version and the galley proof are versions of the publication after peer review.

- The final published version features the final layout of the paper including the volume, issue and page numbers.

Link to publication

\section{General rights}

Copyright and moral rights for the publications made accessible in the public portal are retained by the authors and/or other copyright owners and it is a condition of accessing publications that users recognise and abide by the legal requirements associated with these rights.

- Users may download and print one copy of any publication from the public portal for the purpose of private study or research.

- You may not further distribute the material or use it for any profit-making activity or commercial gain

- You may freely distribute the URL identifying the publication in the public portal.

If the publication is distributed under the terms of Article 25fa of the Dutch Copyright Act, indicated by the "Taverne" license above, please follow below link for the End User Agreement:

www.tue.nl/taverne

Take down policy

If you believe that this document breaches copyright please contact us at:

openaccess@tue.nl

providing details and we will investigate your claim. 


\title{
On the Influence of Phase Noise Induced ICI in MIMO OFDM Systems
}

\author{
Tim C. W. Schenk, Student Member, IEEE, Xiao-Jiao Tao, Member, IEEE, \\ Peter F. M. Smulders, Senior Member, IEEE, and Erik R. Fledderus
}

\begin{abstract}
The influence of transmitter and receiver phase noise (PN) on the performance of a multiple-input multipleoutput orthogonal frequency division multiplexing (MIMO OFDM) based communication system is analyzed. It is shown that in the case of frequency flat Rayleigh fading, the influence of receiver and transmitter $P N$ is equal. In the case of independent Rayleigh fading, however, the impact of the receiver PN is shown to depend on the ratio between the number of transmit and receive branches.
\end{abstract}

Index Terms-MIMO systems, orthogonal frequency division multiplexing (OFDM), phase noise.

\section{INTRODUCTION}

$\mathbf{R}$ ESEARCH concerning multiple-input multiple-output orthogonal frequency division multiplexing (MIMO OFDM) has mainly focussed on systems impaired by additive white Gaussian noise (AWGN). Such analyses will, however, not suffice when implementing a wireless system based on the MIMO OFDM concept. Studies, e.g., [1], concerning the implementation of single-input single-output (SISO) OFDM showed that other system related parameters may significantly affect the system performance. Therefore this letter investigates the influence of non-perfect oscillators, i.e., oscillators causing phase noise (PN), on the performance of a multiple antenna OFDM system. Various papers treat the impact of PN on the performance of SISO OFDM systems, e.g., [2][4]. A common conclusion is that the effect can be split into a multiplicative part, which is equal for all subcarriers and therefore referred to as common phase error (CPE), and an additive part, which is referred to as inter-carrier interference (ICI).

This letter extends previous analyses to take into account the effect of multiple antennas and radio/analog front-ends at both sides of the communication link. Section II shows the impact of PN on the system model. Section III compares the influence of transmitter (TX) and receiver (RX) PN on the performance of a zero-forcing (ZF) based MIMO receiver. This analysis is carried out for two fading cases, i.e., frequency flat fading and per subcarrier independent Rayleigh fading channels. The derived analytical values for the power of the ICI induced estimation error can be used to derive the probability of error of MIMO OFDM systems experiencing PN. Results for more realistic fading situations, with partial correlation between the channel elements for the subcarriers,

Manuscript received December 22, 2004. The associate editor coordinating the review of this letter and approving it for publication was Dr. Sarah Kate Wilson. This work is sponsored by Agere Systems and the Dutch cooperative research project B4 BroadBand Radio@Hand, BTS01063.

Tim C. W. Schenk, Peter F. M. Smulders, and Erik R. Fledderus are with Eindhoven University of Technology, Eindhoven, The Netherlands (e-mail: \{T.C.W.Schenk, P.F.M.Smulders, E.R.Fledderus\}@ @tue.nl).

Xiao-Jiao Tao is with Sony Ericsson Mobile Communications AB, Kista, Sweden (e-mail: Xiao-Jiao.Tao@ sonyericsson.com).

Digital Object Identifier 10.1109/LCOMM.2005.08001. are bounded by the results for these two cases. The analytical results are in Section V compared with experimental results from simulations. The main conclusion is that in the case of independent Rayleigh fading, the impact of the receiver PN depends on the ratio between the number of transmit and receive branches, while this is not the case for frequency flat fading or transmitter PN.

\section{System Model}

Consider a MIMO OFDM system with $N_{t}$ TX and $N_{r}$ RX antennas, denoted here as a $N_{t} \times N_{r}$ system, applying $N_{c}$ subcarriers. It is assumed that the branches at the TX (and similar for the RX branches) use a common oscillator/frequency synthesizer and, thus, experience the same PN process.

As detailed in [5], the received $N_{c} N_{r} \times 1$ frequency domain vector $\hat{\mathbf{x}}$ is given by

$$
\hat{\mathbf{x}}=\left(\mathbf{G}_{\mathrm{RX}} \otimes \mathbf{I}_{N_{r}}\right) \hat{\mathbf{H}}\left(\mathbf{G}_{\mathrm{TX}} \otimes \mathbf{I}_{N_{t}}\right) \hat{\mathbf{s}}+\hat{\mathbf{n}},
$$

where $\mathbf{I}_{N}$ represents the $N \times N$ dimensional identity matrix and $\otimes$ denotes the Kronecker product. The $N_{c} N_{r} \times 1$ transmitted MIMO OFDM vector is given by $\hat{\mathbf{s}}=\left(\mathbf{s}_{1}^{T}, \mathbf{s}_{2}^{T}, \ldots, \mathbf{s}_{N_{c}}^{T}\right)^{T}$, where $\mathbf{s}_{k}$ denotes the $N_{t} \times 1$ frequency domain MIMO transmit vector for the $k$ th subcarrier. We consider a situation where the elements of $\hat{\mathrm{s}}$ are independent, which corresponds to a space division multiplexing system. The quasistatic multipath channel, with an average channel amplitude of unity and delay of zero, is modeled in the frequency domain by the $N_{c} N_{r} \times N_{c} N_{t}$ block diagonal matrix $\hat{\mathbf{H}}$. The $k$ th $N_{r} \times N_{t}$ block diagonal element is $\mathbf{H}_{k}$, the MIMO channel for the $k$ th subcarrier, whose elements we assume to be i.i.d. The $N_{r} N_{c} \times 1$ vector $\hat{\mathbf{n}}$ represents the receiver noise, with i.i.d. zero-mean, complex Gaussian elements with a variance of $\sigma_{n}^{2}$.

The matrices $\mathbf{G}_{\mathrm{TX}}$ and $\mathbf{G}_{\mathrm{RX}}$ model the influence of the TX and RX PN in the baseband system model, respectively. The $(k, l)$ th element of the $N_{c} \times N_{c}$ matrix $\mathbf{G}_{\mathrm{TX}}$, and similarly for $\mathbf{G}_{\mathrm{RX}}$, is given by

$$
g_{q}^{\mathrm{TX}}=\frac{1}{N_{c}} \sum_{i=0}^{N_{c}-1} \exp \left(j \theta_{\mathrm{TX},\left(N_{g}+i+1\right)}\right) \exp \left(-j \frac{2 \pi q i}{N_{c}}\right),
$$

where $q=k-l$, and $\theta_{\mathrm{TX}, n}$ and $\theta_{\mathrm{RX}, n}$ are sampled random variables that represent the transmitter and receiver $\mathrm{PN}$ at sample instant $n$, respectively. Furthermore, $N_{g}$ is the guard interval (GI) length. Note that without PN, $\mathbf{G}_{\mathrm{TX}}$ and $\mathbf{G}_{\mathrm{RX}}$ reduce to identity matrices and (1) becomes the well-known system model for AWGN impaired MIMO OFDM systems. Estimates of the transmitted signal can be found by applying MIMO processing to the received signal $\hat{\mathbf{x}}$.

All elements on the diagonal of $\mathbf{G}_{\mathrm{TX}}$ and $\mathbf{G}_{\mathrm{RX}}$ are equal, i.e., $g_{0}^{\mathrm{TX}}$ and $g_{0}^{\mathrm{RX}}$, respectively, and have unity amplitude. Since they are on the diagonal, they cause a rotation $g_{0}=g_{0}^{\mathrm{RX}} g_{0}^{\mathrm{TX}}$ of 
the wanted signals. As this rotation is equal for all carriers, it is often referred to as common phase error (CPE). The other elements in $\mathbf{G}_{\mathrm{TX}}$ and $\mathbf{G}_{\mathrm{RX}}$ cause interference among carriers, i.e., the ICI $\hat{\boldsymbol{\xi}}$. This property is used to rewrite (1) to $\hat{\mathbf{x}}=$ $g_{0} \hat{\mathbf{H}} \hat{\mathbf{s}}+\hat{\boldsymbol{\xi}}+\hat{\mathbf{n}}$, where

$$
\begin{aligned}
\hat{\boldsymbol{\xi}}=\left(\boldsymbol{\varphi}^{\mathrm{RX}} \otimes\right. & \left.\mathbf{I}_{N_{r}}\right) \hat{\mathbf{H}}\left(\boldsymbol{\varphi}^{\mathrm{TX}} \otimes \mathbf{I}_{N_{t}}\right) \hat{\mathbf{s}}+ \\
& g_{0}^{\mathrm{RX}} \hat{\mathbf{H}}\left(\boldsymbol{\varphi}^{\mathrm{TX}} \otimes \mathbf{I}_{N_{t}}\right) \hat{\mathbf{s}}+g_{0}^{\mathrm{TX}}\left(\boldsymbol{\varphi}^{\mathrm{RX}} \otimes \mathbf{I}_{N_{r}}\right) \hat{\mathbf{H}} \hat{\mathbf{s}},
\end{aligned}
$$

$\varphi^{\mathrm{TX}}=\mathbf{G}_{\mathrm{TX}}-g_{0}^{\mathrm{TX}} \mathbf{I}_{N_{c}}$ and $\varphi^{\mathrm{RX}}$ has a similar structure as $\varphi^{\mathrm{TX}}$. For the remainder of the analysis, we assume the CPE $g_{0}$ can be perfectly removed, e.g., using the method proposed in [5], so the degradation in performance is only caused by the ICI term.

\section{INFLUENCE OF ICI ON THE ESTIMATED SYMBOLS}

When the number of carriers is chosen to be large in comparison to the size of the channel dispersion, the MIMO processing can be applied per subcarrier, since the channel experienced at a subcarrier can be regarded frequency flat. Here zero-forcing (ZF) processing is considered, which multiplies the received signal vector with the pseudo-inverse of the estimated channel matrix. For convenience the channel matrix is assumed to be perfectly known at the RX.

In case of PN at both TX and RX, the estimated version of the transmitted signal $\tilde{\mathbf{s}}$ after ZF processing is given by

$$
\tilde{\mathbf{s}}=g_{0} \hat{\mathbf{s}}+\hat{\mathbf{H}}^{\dagger} \hat{\boldsymbol{\xi}}+\hat{\mathbf{H}}^{\dagger} \hat{\mathbf{n}}
$$

where $^{\dagger}$ denotes the pseudo-inverse of a matrix. The order in which the TX and RX term appear in (3), indicate that TX and RX PN induced ICI differently influence the performance of the estimator. To compare their influence, they are analyzed separately in the remainder of this letter.

\section{A. Transmitter Phase Noise}

For the case of only TX PN and where, thus, the RX oscillator is ideal, i.e., $\mathbf{G}_{\mathrm{RX}}=\mathbf{I}_{N_{c}}$, the term $\hat{\mathbf{H}}^{\dagger} \hat{\boldsymbol{\xi}}$ represents the ICI induced error in the estimated TX signal and is given by $\hat{\mathbf{H}}^{\dagger} \hat{\boldsymbol{\xi}}=\left(\boldsymbol{\varphi}^{\mathrm{TX}} \otimes \mathbf{I}_{N_{t}}\right) \hat{\mathbf{s}}$. This error term for the $k$ th carrier $\mathbf{\Xi}_{k}$ is given by $\sum_{i=1, i \neq k}^{N_{c}} g_{k-i}^{\mathrm{TX}} \mathbf{s}_{i}$. The power of this error term averaged over $N_{t}$ antennas for the $k$ th carrier is then given by

$$
P_{\mathbf{\Xi}_{k}}=\frac{1}{N_{t}} \mathcal{E}\left\{\boldsymbol{\Xi}_{k}^{H} \boldsymbol{\Xi}_{k}\right\}=\sigma_{s}^{2} \sum_{i=1, i \neq k}^{N_{c}} \mathcal{E}\left\{\left|g_{k-i}^{\mathrm{Tx}}\right|^{2}\right\},
$$

where ${ }^{H}$ denotes the conjugate transpose and the elements of $\mathrm{s}$ are assumed to be i.i.d. distributed with zero-mean and a variance of $\sigma_{s}^{2}$.

\section{B. Receiver Phase Noise}

For a system only experiencing RX PN, i.e., $\mathbf{G}_{\mathrm{TX}}=\mathbf{I}_{N_{c}}$, the term $\hat{\mathbf{H}}^{\dagger} \hat{\boldsymbol{\xi}}$ represents the ICI induced error in the estimated TX signal and is given by $\hat{\mathbf{H}}^{\dagger} \hat{\boldsymbol{\xi}}=\hat{\mathbf{H}}^{\dagger}\left(\varphi^{\mathrm{RX}} \otimes \mathbf{I}_{N_{r}}\right) \hat{\mathbf{H}} \hat{\mathbf{s}}$. This error term for the $k$ th carrier $\boldsymbol{\Xi}_{k}$ is given by $\mathbf{H}_{k}^{\dagger} \sum_{i=1, i \neq k}^{N_{c}} g_{k-i}^{\mathrm{RX}} \mathbf{H}_{i} \mathbf{s}_{i}$. The average power of $\boldsymbol{\Xi}_{k}$ is given by

$$
\begin{aligned}
& P_{\mathbf{\Xi}_{k}}=\frac{1}{N_{t}} \mathcal{E}\left\{\boldsymbol{\Xi}_{k}^{H} \boldsymbol{\Xi}_{k}\right\} \\
& =\frac{\sigma_{s}^{2}}{N_{t}} \sum_{i=1, i \neq k}^{N_{c}} \mathcal{E}\left\{\left|g_{k-i}^{\mathrm{RX}}\right|^{2}\right\} \operatorname{tr}\left[\mathcal{E}\left\{\mathbf{H}_{i}^{H} \mathbf{H}_{k}^{\dagger H} \mathbf{H}_{k}^{\dagger} \mathbf{H}_{i}\right\}\right] .
\end{aligned}
$$

The average power of the error term of estimated symbols depends on the interaction between channel elements at different subcarrier locations. Therefore, the channel conditions will influence the power of the error term. To quantify the impact, the analysis is carried out for two special cases: Case 1, where the channel is flat Rayleigh faded over the whole system bandwidth; and Case 2, where all subcarriers experience independent Rayleigh faded channel elements.

1) Case 1 - Flat Fading: For the flat fading case $\mathbf{H}_{k}=\mathbf{H}_{i}$, and (6) can, thus, be simplified to

$$
P_{\Xi_{k}}=\sigma_{s}^{2} \sum_{i=1, i \neq k}^{N_{c}} \mathcal{E}\left\{\left|g_{k-i}^{\mathrm{RX}}\right|^{2}\right\} .
$$

When comparing (7) to (5), it is clear that for flat fading there is no difference between the impact of TX and RX PN.

2) Case 2 - Independent Fading: For the independent fading case the elements of $\mathbf{H}_{k}$ and $\mathbf{H}_{l}$ are independent and identical distributed according to the zero-mean, unit variance complex Gaussian distribution. Using these channel properties,

(6) can be rewritten to

$$
\begin{aligned}
P_{\Xi_{k}} & =\frac{\sigma_{s}^{2}}{N_{t}} \sum_{i=1, i \neq k}^{N_{c}} \mathcal{E}\left\{\left|g_{k-i}^{\mathrm{RX}}\right|^{2}\right\} \operatorname{tr}\left[\mathcal{E}\left\{\left[\mathbf{H}_{k} \mathbf{H}_{k}^{H}\right]^{\dagger}\right\} \mathcal{E}\left\{\mathbf{H}_{i} \mathbf{H}_{i}^{H}\right\}\right] \\
& =\sigma_{s}^{2} \operatorname{tr}\left[\mathcal{E}\left\{\left[\mathbf{H}_{k} \mathbf{H}_{k}^{H}\right]^{\dagger}\right\}\right] \sum_{i=1, i \neq k}^{N_{c}} \mathcal{E}\left\{\left|g_{k-i}^{\mathrm{RX}}\right|^{2}\right\}
\end{aligned}
$$

where $\left[\mathbf{H}_{k} \mathbf{H}_{k}^{H}\right]^{\dagger}$ is distributed according to the complex inverse Wishart distribution [6]. For the special case of $N_{r}>N_{t}$, the authors of [6] show that $\operatorname{tr}\left[\mathcal{E}\left\{\left[\mathbf{H}_{k} \mathbf{H}_{k}^{H}\right]^{\dagger}\right\}\right]=$ $N_{t} /\left(N_{r}-N_{t}\right)$. When we apply this, (8) is simplified to

$$
P_{\Xi_{k}}=\frac{N_{t}}{N_{r}-N_{t}} \sigma_{s}^{2} \sum_{i=1, i \neq k}^{N_{c}} \mathcal{E}\left\{\left|g_{k-i}^{\mathrm{RX}}\right|^{2}\right\} .
$$

When comparing the result of (9) with the one for TX PN in (5), we can conclude that when $N_{r}>2 N_{t}$, the RX PN has less impact than TX PN for independent Rayleigh faded channels. When $N_{t}<N_{r}<2 N_{t}$, the RX PN has greater impact than TX PN. For example, for a 2 TX and 3 RX system, $N_{t} /\left(N_{r}-N_{t}\right)=2$, RX PN leads to a twice greater average power level of the error term.

\section{Phase Noise Model: Sampled Brownian Motion}

The average power of $\boldsymbol{\Xi}_{k}$, as derived above, depends on the PN process through $g_{q}$. Here we define it to be a sampled Brownian motion, a commonly used model for free-running oscillators, which is given by $\theta_{\mathrm{TX}, n}=\sum_{i=0}^{n} \varepsilon_{n}$, where $\varepsilon_{n} \sim$ $\mathcal{N}\left(0, \sigma_{\varepsilon}^{2}\right)$ and $\sigma_{\varepsilon}^{2}=4 \pi \beta T$. Here $\beta$ denotes the one-sided $-3 \mathrm{~dB}$ bandwidth of the corresponding Lorentzian spectrum and $T$ is the sample time. Applying the properties of the PN process, $\mathcal{E}\left\{\left|g_{q}\right|^{2}\right\}$ can be calculated. Hereto $g_{q}$, as defined in (2), is rewritten for $q \in\left\{-N_{c}+1, \ldots,-1,1, \ldots, N_{c}-1\right\}$ using the small angle approximation, which is valid when the $-3 \mathrm{~dB}$ linewidth $\beta$ is small as compared to the subcarrier spacing $1 /\left(T N_{c}\right)$. When, furthermore, the order of summation is changed, $g_{q}$ is given by

$$
g_{q}=\frac{j \eta}{N_{c}} \sum_{n=0}^{N_{c}-1} \varepsilon_{n} \sum_{i=0}^{n} \exp \left(-j 2 \pi q n / N_{c}\right)
$$


where $\eta=\exp \left(j \sum_{l=0}^{N_{g}+1} \varepsilon_{l}\right)$. The expected value of the power of $g_{q}$ can now be rewritten to

$$
\mathcal{E}\left\{\left|g_{q}\right|^{2}\right\}=\frac{\sigma_{\varepsilon}^{2} \sum_{n=0}^{N_{c}-1} \sin ^{2}\left(\frac{q n \pi}{N_{c}}\right)}{N_{c}^{2} \sin ^{2}\left(q \pi / N_{c}\right)}=\frac{2 \pi \beta T}{N_{c} \sin ^{2}\left(\frac{q \pi}{N_{c}}\right)} .
$$

Substituting expression (11) into (5), for the case of TX PN, the expression for the average power of the error term due to ICI on the $k$ th carrier can be written as

$$
P_{\Xi_{k}}=\frac{2 \sigma_{s}^{2} \pi \beta T}{N_{c}^{2}} \sum_{i=1, i \neq k}^{N_{c}} \sin ^{-2}\left(\frac{(k-i) \pi}{N_{c}}\right) .
$$

In most systems the number of carriers is chosen to be a power of 2, i.e., $N_{c}=2^{M}$, due to ease of implementation of the discrete Fourier transform (DFT) for this number of carriers. When this is assumed, the sum in (12) can be written as a recursive expression and it is found that it can be rewritten to

$$
\begin{gathered}
\sum_{i=1, i \neq k}^{2^{M}} \sin ^{-2}\left(\frac{(k-i) \pi}{2^{M}}\right)=\sum_{i=1}^{2^{M}-1} \sin ^{-2}\left(\frac{i \pi}{2^{M}}\right) \\
=1+2 \sum_{i=1}^{2^{M-1}-1} \sin ^{-2}\left(\frac{i \pi}{2^{M}}\right)=\frac{2^{2 M}-1}{3} .
\end{gathered}
$$

Substituting (13) in (12) results in the following expression for the average power of the error term $\boldsymbol{\Xi}_{k}$ :

$$
P_{\Xi_{k}}=\frac{2 \sigma_{s}^{2} \pi \beta T\left(N_{c}^{2}-1\right)}{3 N_{c}}
$$

For a large number of carriers this is well approximated by $\frac{2}{3} \sigma_{s}^{2} \pi \beta T N_{c}$. Note that this expression no longer depends on the carrier index $k$.

Similarly, the expressions for the average ICI power in (7) and (9) can be further simplified if the PN is modeled by a sampled Brownian motion. The approximation of $P_{\Xi_{k}}$ for a large number of carriers for Case I, i.e., flat Rayleigh fading, is given by $\frac{2}{3} \sigma_{s}^{2} \pi \beta T N_{c}$ and for Case II, i.e., independent Rayleigh fading, by $\frac{2 N_{t}}{3\left(N_{r}-N_{t}\right)} \sigma_{s}^{2} \pi \beta T N_{c}$.

\section{NUMERICAL RESUlts}

The analytical results of Section IV are in Fig. 1 compared with simulation results from Monte-Carlo simulations. In these simulations the sample time $T$ was $50 \mathrm{~ns}$, the number of subcarriers $N_{c}=64,64-\mathrm{QAM}$ modulation was applied and $\sigma_{s}^{2}=1$. The PN is modeled as a sampled Brownian motion. In Fig. 1 the average ICI induced estimation error is plotted as function of the $-3 \mathrm{~dB}$ bandwith of the Lorentzian power spectral density of the PN $\beta$. The results are given for different combinations of number of TX and RX antennas. Every subcarrier experienced an i.i.d. Rayleigh faded channel in the simulations.

It is clear from Fig. 1 that there is good agreement between the analytical results from Section IV and the simulation results. The discrepancy at high $\beta$ values can be explained by the fact that the small angle approximation is no longer valid. Here $\beta$ is no longer small compared to the subcarrier spacing, which is $312.5 \mathrm{kHz}$ in this case.

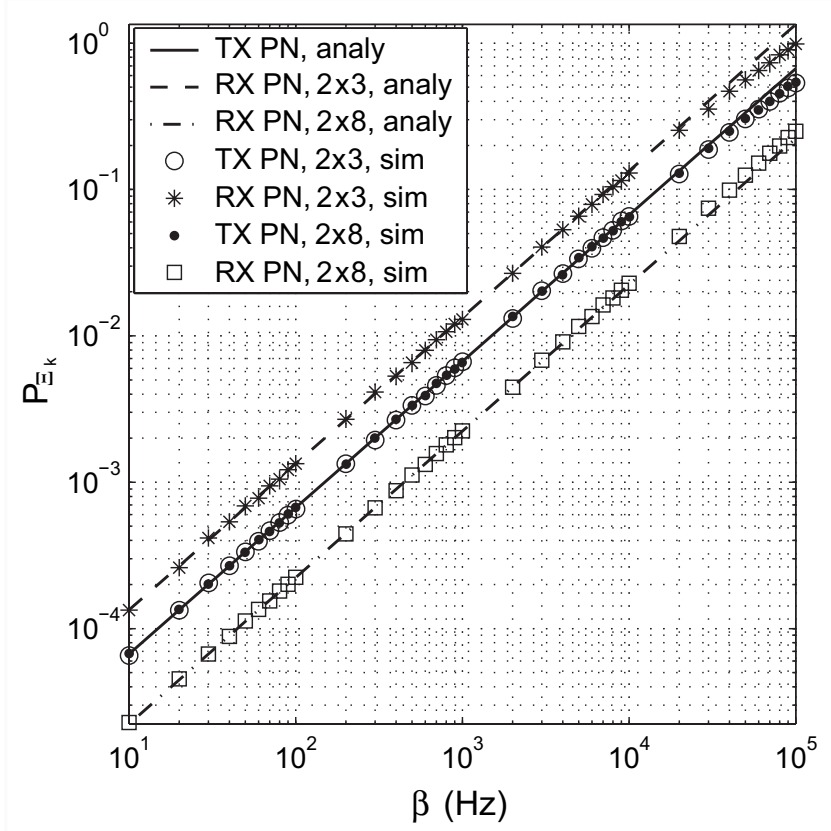

Fig. 1. Average power of error in estimated symbols due to inter-carrier interference as function of the PN corner frequency $\beta$, for $T=50 \mathrm{~ns}$, $N_{c}=64, \sigma_{s}^{2}=1,64-\mathrm{QAM}$ modulation. Channel is modeled as independent Rayleigh faded per subcarrier. Analytical (analy) and simulation (sim) results are depicted.

\section{Vi. CONCLUSIONS}

In this letter the impact of phase noise (PN) on the performance of a wireless system combining multiple-input multiple-output (MIMO) with orthogonal frequency division multiplexing (OFDM) is examined. The influence of transmitter and receiver phase noise is shown to be different. An expression is found for the power of the error term after zeroforcing estimation caused by the ICI term. For TX PN the power of this error term is equal for all MIMO configurations. The power of the error term for RX PN is equal to the TX case in the case of a flat Rayleigh faded channel. For the case of independent Rayleigh faded channels, however, the RX PN is concluded to have less impact than TX PN when $N_{r}>2 N_{t}$ and more impact when $N_{t}<N_{r}<2 N_{t}$. Simulation results confirm the results from the analytical study.

\section{REFERENCES}

[1] B. Come, R. Nessand, S. Donnay, L. van der Perre, W. Eberle, P. Wambacq, M. Engels, and I. Bolsens, "Impact of front-end nonidealities on bit error rate performance of WLAN-OFDM transceivers," in Proc. IEEE Radio and Wireless Conference 2000, pp. 91-94.

[2] T. Pollet, M. van Bladel, and M. Moeneclaey, "BER sensitivity of OFDM systems to carrier frequency offset and Wiener phase noise," IEEE Trans. Commun., vol. 43, pp. 191-193, Feb./Mar./Apr. 1995.

[3] S. Wu and Y. Bar-Ness, "Performance analysis on the effect of phase noise in OFDM systems," in Proc. IEEE Seventh International Symposium on Spread Spectrum Techniques and Applications, 2002, vol. 1, pp. 133-138.

[4] D. Petrovic, W. Rave, and G. Fettweis, "Performance degradation of coded-OFDM due to phase noise," in Proc. 57th IEEE VTC 2003-Spring, Apr. 2003, vol. 2, pp. 1168-1172.

[5] T. C. W. Schenk, X.-J. Tao, P. F. M. Smulders, and E. R. Fledderus, "Influence and suppression of phase noise in multi-antenna OFDM," in Proc. 60th IEEE VTC 2004-Fall, vol. 2, pp. 1443-1447, Sept. 2004.

[6] D. Maiwald and D. Kraus, "Calculation of moments of complex wishart and complex inverse wishart distributed matrices," IEE Proc. Radar, Sonar Navig., vol. 147, pp. 162-168, Aug. 2000. 